\title{
Transmission Electron Microscopy Study of Nonpolar a-Plane GaN Grown by Pendeo-Epitaxy on (1120) 4H-SiC.
}

\author{
D.N. Zakharov ${ }^{1}$, Z. Liliental-Weber ${ }^{1}$, B. Wagner ${ }^{2}$, Z.J. Reitmeier ${ }^{2}$, E.A. Preble ${ }^{2}$, and R.F. \\ Davis $^{2}$ \\ ${ }^{1}$ Lawrence Berkeley National Laboratory, MS 62-203, Berkeley, CA 94720 \\ ${ }^{2}$ North Carolina State University, Raleigh, NC 27695
}

\begin{abstract}
Pendeo-epitaxy has been applied to nonpolar a-plane GaN layers in order to observe if such process will lead to defect reduction in comparison with direct growth on this plane. Uncoalesced and coalesced a-plane GaN layers with thicknesses $2 \mu \mathrm{m}$ and $12 \mu \mathrm{m}$, respectively have been studied by conventional and high resolution electron microscopy. The following structural defects have been observed in pendeo-epitaxial layers: (1) basal stacking faults, (2) threading dislocations and (3) prismatic stacking faults. Drastic decrease of threading dislocation density and stacking faults have been observed in 'wing' areas with respect to 'seed' areas. Cross-section images reveal cracks and voids at the areas where two coalesced wings meet each other. High resolution electron microscopy shows that the majority of stacking faults are low-energy planar defects of the types $\mathrm{I}_{1}, \mathrm{I}_{2}$ and $\mathrm{I}_{3}$. The $\mathrm{I}_{3}$ type basal stacking fault, predicted theoretically, has been observed experimentally for the first time.
\end{abstract}

\section{INTRODUCTION}

Recent studies have shown that spontaneous polarization and piezoelectric effect can be eliminated in GaN grown along non-polar directions, such as [11이 and [1 100$]$ $[1,2]$. These two effects were observed in GaN layers grown along [0001] direction and led to high interface charge densities and spatial separations of electron and holes wave functions in GaN-based quantum well structures [3]. Transmission electron microscopy investigations performed on (1100) (m-plane) and (1120) (a-plane) GaN layers grown by heteroepitaxial methods on different substrates revealed, however, high density of basal stacking faults (BSFs) and associated with them threading dislocations [4-6]. Since Pendeoepitaxy (PE) was proven to be effective method for defects reduction in GaN [7], we applied this method for a-plane GaN grown by metalorganic vapor phase epitaxy on (1120) $4 \mathrm{H}-\mathrm{SiC}$ substrates with AlN buffer layers. Uncoalesced and coalesced samples have been produced. These samples have been studied by transmission electron microscopy (TEM) in cross-section and plan-view configuration in order to investigate structural defects present in the layers and detect possible defects reduction.

\section{EXPERIMENTAL}

The epitaxial AlN buffer layers, the subsequently deposited epitaxial GaN seed films and the pendeo-epitaxial (PE) GaN layers were grown in-situ in a cold-wall, vertical, 
pancake-style metalorganic vapor phase epitaxy (MOVPE) system. These PE layers were grown on $4 \mathrm{H}-\mathrm{SiC}(11 \underline{20})$ substrates previously etched in a $10 \% \mathrm{HF}$ solution in order to remove the native oxide. The $100 \mathrm{~nm}$ thick AlN buffer layers and the $500 \mathrm{~nm}$ thick GaN seed layers were grown at 20 Torr and temperatures of $1100^{\circ} \mathrm{C}$ and $1020^{\circ} \mathrm{C}$, respectively. The precursor species (mass flow rates) of tri-methylaluminum (TMA; $5.4 \mu \mathrm{mol} / \mathrm{min}$ carried in $\mathrm{H}_{2}$ ), tri-ethylgallium (TEG; $101 \mu \mathrm{mol} / \mathrm{min}$ carried in $\mathrm{H}_{2}$ ) and ammonia $\left(\mathrm{NH}_{3}\right.$; $0.14 \mathrm{~mol} / \mathrm{min}$ ) were mixed with a high-purity $\mathrm{H}_{2}$ diluent $(3 \mathrm{slm})$ in a two-inch internal diameter sleeve located approximately five centimeters above the substrate.

A Ni layer was deposited on patterned photoresist stripes on each GaN seed layer by e-beam evaporation. Etch mask stripes were subsequently produced using standard photolithography lift-off techniques. An inductively coupled plasma (ICP) system and a mixture of $\mathrm{Cl}_{2}$ and $\mathrm{BCl}_{3}$ gases at a 9:1 ratio were used to etch the unmasked striped regions through the $\mathrm{GaN}$ and the AlN layers and into the near-surface regions of the $\mathrm{SiC}$ substrate. The $\mathrm{Ni}$ was then removed using a $5 \mathrm{~min}$ dip in $50 \% \mathrm{HNO}_{3}$. The remaining $\mathrm{GaN}$ seed layer consisted of $2 \mu \mathrm{m}$ wide stripes oriented along [1100] direction separated from each other by $3 \mu \mathrm{m}$. The exposed (0001) sidewalls and the top (1120) faces of the stripes were subsequently dipped in $50 \%$ hot $\mathrm{HCl}$ to clean the surface prior to regrowth of the $\mathrm{PE}$ layer.

Samples were studied by conventional transmission electron microscopy (TEM) and high resolution electron microscopy (HREM) using a JEOL 3010 and Philips CM 300 microscopes, respectively. Both microscopes operated at $300 \mathrm{kV}$. Cross-sectional and planview samples for these studies were prepared by mechanical polishing followed by dimpling down to $10 \mu \mathrm{m}$ and further Ar ion milling at $5 \mathrm{kV}$ until perforation occurred.

\section{RESULTS AND DISCUSSION}

\section{A. Samples morphology.}

Figure 1a shows cross-section bright field image of an uncoalesced a-plane PE layer. One can notice different sizes of the 'wings' on the left and the right sides of the 'seed' area. Similar PE layers grown with [0001] growth direction have both 'wings' of the same size [6]. This difference in size results from the fact that our PE layers start to overgrow in [0001] direction on the left side of the 'seed' and in [0001] direction on the right side of the 'seed'. The growth rate for these two directions ( $\mathrm{N}$ - and Ga-polarity) is different [7] and can be estimated from this micrograph. Based on our micrographs the growth with N-polarity (Figure 1b) is about 17 times slower then growth with Ga-polarity.

The top surface of the left 'wing' and major part of the top surface of the 'seed' are flat and are aligned on (1120) plane of the wurtzite structure of GaN (Figure 1a). However, a part of the surface of the 'seed' and the surface of the right 'wing' are truncated. The truncated surface is imaged egde-on along [1100] zone axis. This surface makes $58^{\circ} \pm 1^{\circ}$ angle with (0001) plane and $32^{\circ} \pm 1^{\circ}$ angle with (1120) plane of GaN, and can be described as (112) plane.

Figure 2 a shows cross-sectional bright field image of a coalesced sample. The surface of the sample is flat. The sample thickness is $12 \mu \mathrm{m}$. The stripe width and the stripe period are $2 \mu \mathrm{m}$ and $5 \mu \mathrm{m}$, respectively. The image, however, reveals voids at areas where two coalesced wings meet each other (meeting fronts (MFs), marked by dashed double- 


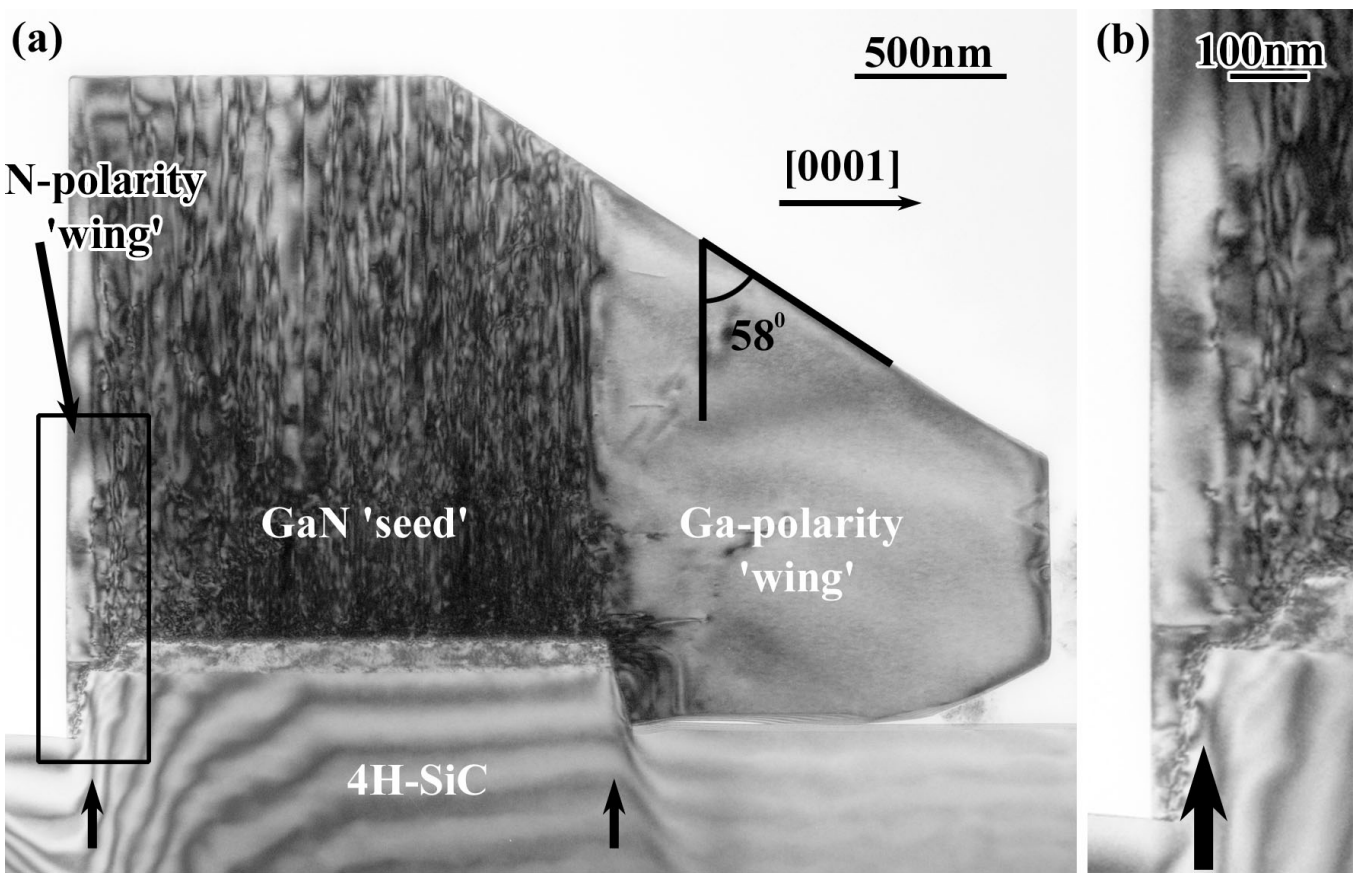

Figure 1. (a) Bright field cross-section image of an uncoalesced a-plane PE sample grown on (11 20$)$ 4H-SiC taken along [1100] zone axis. Approximate position of 'wings'/'seed' boundaries are indicated by two black arrows. Inclined surface of a part of the 'seed' and the Ga-polarity 'wing' makes $58^{\circ} \pm 1^{\circ}$ angle with the c-plane of the wurtzite GaN. (b) Magnified part of the image (a) marked by black box shows boundary between N-polarity 'wing' and 'seed'. The boundary shows itself in drastic decrease in number of threading dislocations associated with basal stacking faults.

head arrows). Cracks are also often observed at MFs (marked by arrow in Figure 2a). These cracks and voids are an indirect way to indicate meeting fronts of two adjacent 'wings'.

\section{B. Basal stacking faults and threading dislocations.}

High density of stacking faults formed on the basal plane of hexagonal GaN was observed in cross-sectional TEM images of the PE layers recorded from 'seed' areas. These basal stacking faults (BSFs) are visible in bright field image obtained under two beam condition with $g=1100$ as thin lines arranged perpendicular to the c-direction of the wurtzite $\mathrm{GaN}$ (Figure 2b). The measured density of SFs is $\sim 1.3 \times 10^{6} \mathrm{~cm}^{-1}$. Figure 2c represent bright field image of a 'wing' area obtained with the same two beam condition as the Figure $2 \mathrm{~b}$. One can notice drastic decrease in density of BSFs in the 'wing' areas in comparison to the 'seed' areas. Two BSFs are marked by arrows on the Figure 2c. Average density of BSFs measured over number of 'wing' areas is $\sim 1.2 \times 10^{4} \mathrm{~cm}^{-1}$.

BSFs are also visible in samples prepared in plan-view configuration. Our diffraction contrast experiments have shown that BSFs are in strong contrast for $g=1100$ (Figure 3a), whereas for $\boldsymbol{g}=0002$ contrast from BSFs vanishes (Figure 3b). Some of BSFs end inside the GaN crystal (examples are marked by black arrows in Figure 3a) and must be terminated by dislocations. These dislocations with nonzero [0001] component of a Burgers 

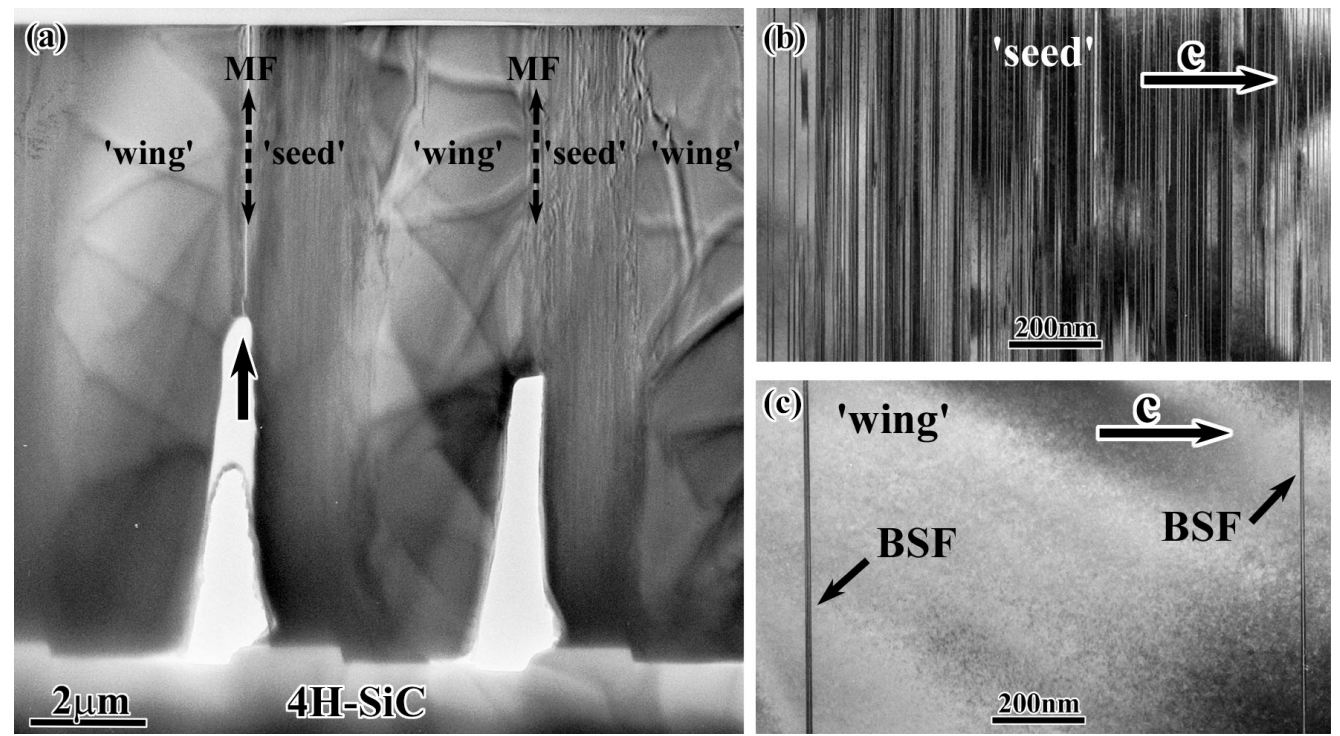

Figure 2. (a) Cross-sectional TEM image of a coalesced a-plane pendeo-epitaxial $\mathrm{GaN}$ layer grown on (1120) 4H-SiC substrate. Cracks (marked by arrow) are often observed at meeting fronts (MF) of two adjacent 'wings'. Distribution of basal stacking faults in the 'seed' (b) and in the 'wing' areas (c) is shown with higher magnification. Note decrease of basal stacking fault densities in the 'seed' area in comparison to the 'wing' area.

vector terminating BSFs are in strong contrast in Figure $3 \mathrm{~b}$ (marked by black arrows). The density of dislocations reduces by more than two orders of magnitude from $\sim 4.2 \times 10^{10} \mathrm{~cm}^{-2}$ in 'seed' areas to $\sim 1.0 \times 10^{8} \mathrm{~cm}^{-2}$ in 'wing' areas.

High resolution transmission electron microscopy (HREM) was applied to determine the nature of BSFs. Figure 4 shows characteristic HREM images of BSFs observed in our samples. Analysis of these images indicates that BSFs change perfect ...ABABAB... stacking sequence into the ...ABABCBC... (Figure 4a), the ...ABABCACA... (Figure $4 \mathrm{~b}$ ), and the ...ABABCBABA... (Figure 4c), respectively. The first two sequences correspond to $I_{1}$ and $I_{2}$ types of basal stacking faults [8]. These BFSs have to be bounded by partial dislocations with Burgers vectors $\boldsymbol{b}=1 / 6<2023>$ and $\boldsymbol{b}=1 / 3<1100>$, respectively. The last sequence corresponds to the $\mathrm{I}_{3}$ type BSF observed only in the 'seed' areas. This type of faults was predicted theoretically and has been shown to have the second lowest formation energy after $I_{1}$ type BSF [9]. It appears to us that it is a first experimental indication of their presence in $\mathrm{GaN}$.

\section{Prismatic stacking faults.}

In addition to BSFs formed on c-planes a different type of faults have been observed on prismatic planes of wurtzite GaN in 'seed' and 'wing' areas of studied a-plane PE GaN layers. It is possible to determine fault vectors of these prismatic stacking faults (PSFs) by observing under which two beam condition the contrast from the PSF is vanished. Figure 5a shows a bright field image of a 'wing' area obtained under two beam condition with $\boldsymbol{g}=1 \underline{100}$. The image shows a contrast from two BSFs intersecting with a PSF. The same PSF is out of contrast for images taken with $g=2110$, and $g=1 \underline{101}$, respectively (Figures $5 \mathrm{~b}$ and 5c). Taking also into account that PSFs produce a contrast on images taken with 


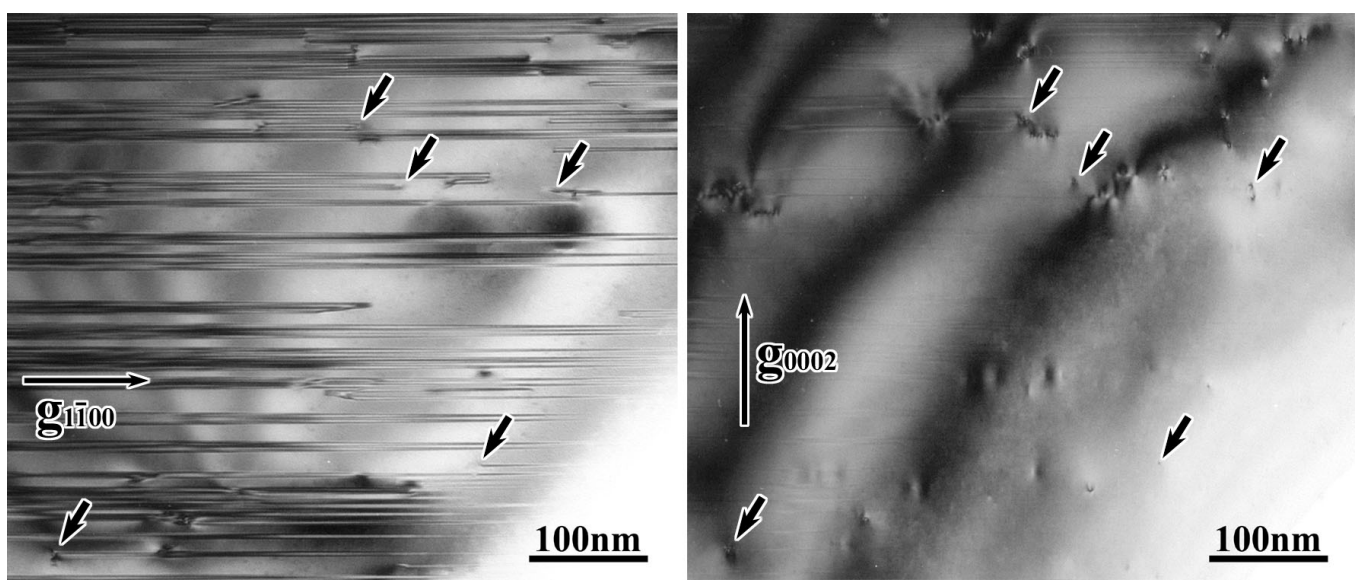

Figure 3. Bright field TEM images of a plan-view sample ('seed' area) obtained with two perpendicular $g$-vectors: (a) $g=1100$; parallel lines represent stacking faults formed on the basal plane of $\mathrm{GaN}$. (b) $\boldsymbol{g}=0002$; partial dislocations having a nonzero [0001] component of a Burgers vector are in contrast. Black arrows in both images show corresponding places of basal stacking faults terminated by dislocations.
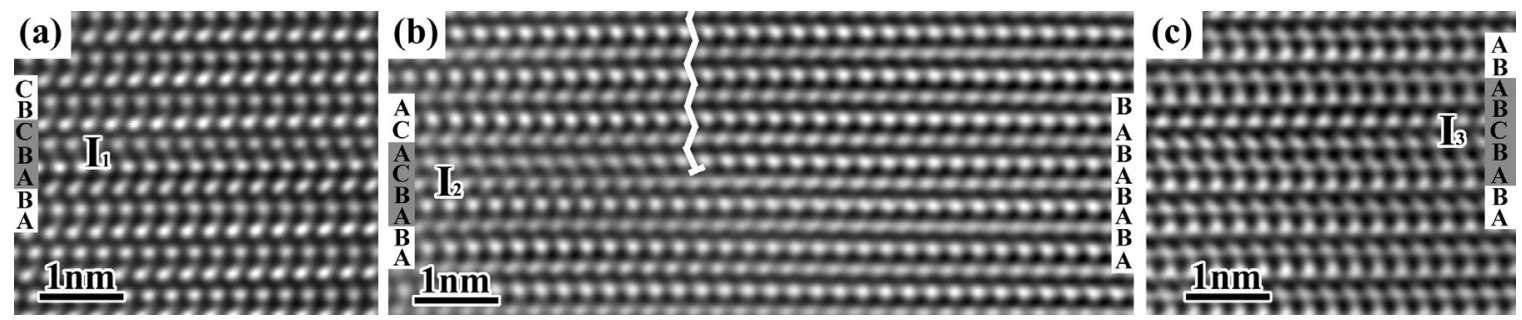

Figure 4. High resolution images of a plan-view sample shows typical stacking faults observed in the sample: (a) $\mathrm{I}_{1}$ type with stacking sequence ...ABABCBC...; (b) $\mathrm{I}_{2}$ type with stacking sequence ...ABABCACA... Shockley partial dislocation $(\boldsymbol{b}=1 / 3<1 \underline{100>})$, which terminates the $I_{2}$ basal stacking fault is marked in (b); (c) $I_{3}$ type with stacking sequence ...AB ABCBABA....
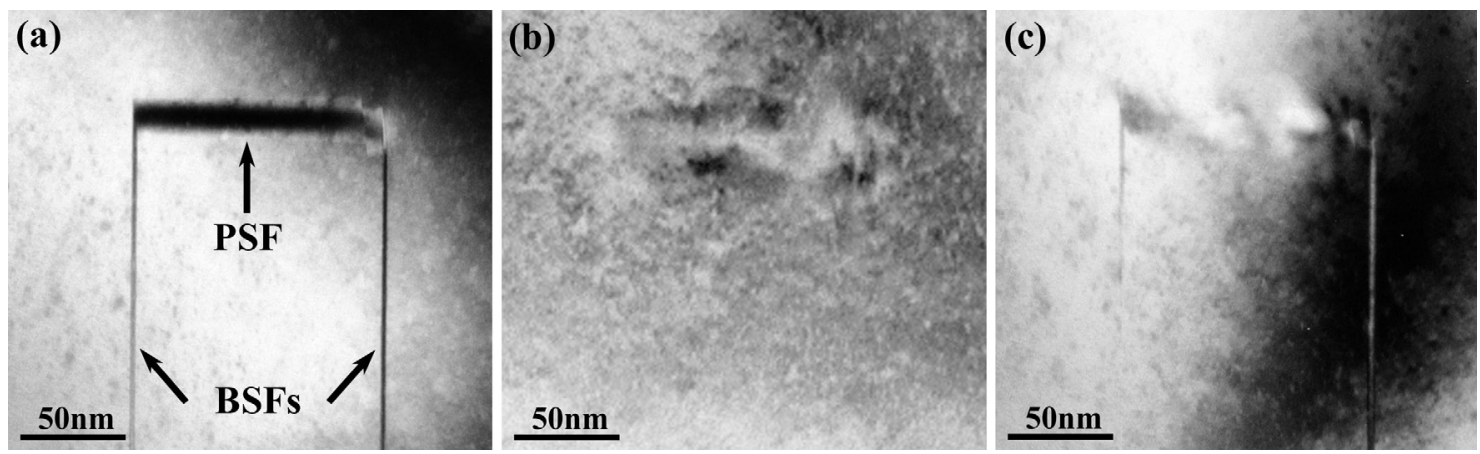

Figure 5. Bright field images of two basal stacking faults intersecting with a prismatic stacking fault obtained under different two beam conditions: (a) $g=1 \underline{100}$, both basal stacking faults and prismatic stacking fault are in contrast. Prismatic stacking fault is out of contrast for (b) $g=0002$, (c) $g=2110$ and (d) $g=1 \underline{101}$, respectively. 
$\boldsymbol{g}=3 \underline{3} 00$, but not with $\boldsymbol{g}=0002$ and $\boldsymbol{g}=2 \underline{2} 00$ (not shown here) their displacement vector should be 1/2[0111] [10]. PSFs are always found to intersect with $\mathrm{I}_{1}$ type BSFs. As soon as fault vectors of a PSF and a BSF are not equal, stair-rod dislocations are expected at such intersections.

\section{SUMMARY}

Uncoalesced and coalesced pendeo-epitaxial a-plane GaN layers grown on (1120) 4H-SiC substrate with AlN buffer layer have been investigated by conventional and high resolution electron microscopy. Samples showed the presence of low energy $I_{1}, I_{2}$ and $I_{3}$ types of basal stacking faults, threading dislocations and stacking faults formed on prismatic planes of the wurtzite GaN structure. The $\mathrm{I}_{3}$ type BSF having second lowest formation energy after $\mathrm{I}_{1}$ type BSF were observed experimentally for the first time. Drastic decrease in BSFs and threading dislocations densities revealed in 'wing' areas in comparison to 'seed' areas.

\section{ACKNOLEDGMENTS}

This work was supported the Air Force Office of Scientific Research (Dr. G. Witt) under the Order No. OGMORD 52K44134. The use of the facilities at the National Center for Electron Microscopy at Lawrence Berkeley National Laboratory is greatly appreciated.

\section{REFERENCES}

1. P. Waltereit, O. Brandt, A. Trampert, H. T. Grahn, J. Menniger, M. Ramsteiner, M. Reiche, and K. H. Ploog, Nature 406, 865 (2000)

2. M. D. Craven, P. Waltereit, W. Feng, J. S. Speck, and S. P. DenBaars, Jap. J. Appl. Phys. Lett. 42, L235 (2003)

3. F. Bernardini and V. Fiorentini: Phys. Rev. B 57, 9427 (1998)

4. T.Y. Liu, A. Trampert, Y.J. Sun, O. Brandt, K.H. Ploog, Phil. Mag. Lett. 84, 435 (2004)

5. M.D. Craven, S.H. Lim, F. Wu, J.S. Speck and S.P. DenBaars, Appl. Phys. Lett. 81, 469 (2002)

6. D.N. Zakharov, Z. Liliental-Weber, B. Wagner, Z.J. Reitmeier, E.A. Preble, and R.F. Davis, Mat. Res. Symp. Proc. 798, 747 (2004)

7. P. Gibart, Rep. Prog. Phys. 67, 667 (2004)

8. D. Hull and D.J. Bacon, Introduction to Dislocation, $3^{\text {rd }}$ Ed., Intl. Series on Materials Science and Technology, (Oxford, New York, Beijing, Frankfurt, Sao Paulo, Sydney, Tokyo, Toronto: Pergamon Press, 1984), p.112

9. C. Stampfl and C.G. Van de Walle, Phys. Rev. B 57, R15052 (1998)

10. C.M. Drum, Phil. Mag. 11, 313 (1965) 